\title{
The Evolution and Impact of Acute Stroke Units on Outcome in Stroke Patients
}

\author{
John W. Norris, Vladimir Hachinski
}

Can. J. Neurol. Sci. 2009; 36: 274-276

\section{Early concepts of intensive stroke care}

Stroke and cerebrovascular disease remained a neglected and unexplored subject until the late 1960s, while progress in knowledge of other vascular disorders such as heart disease grew exponentially. The reason was simply that 'stroke' was believed to be a hopeless and incurable disorder with no conceivable future therapy. The then current term "cerebrovascular accident" (CVA) replaced labelling for understanding and invited confusion between the sides of the brain or of the body involved. "Left sided CVA" meant a left hemiplegia to some and a left brain lesion to others. More often than not, "CVA" stood for "confused vascular analysis".

Mainly due to an initiative from the Institute of Neurological Diseases and Blindness, in Washington, funds were allocated to a newly established US stroke research program. This stimulated the initial concept of stroke intensive care units (SICU) first developed in the US, using the parallel model of Acute Coronary Care Units, which were already in operation throughout the country and clearly effective. The first published account of a SICU in Pittsburgh, by a team of internal medicine physicians and nurses, and so the worlds first stroke unit, was established in $1966 .^{1}$ However, the authors considered the results disappointing and expectations of results in stroke patients, similar to those found in coronary care units, could not be equated, since early stroke deaths were due to stroke itself and would have to 'await new developments in the medical therapy of stroke'.

Nevertheless, the perceived similarity of heart disease and stroke caused the brief establishment of combined 'Heart-Stroke' units, staffed by cardiologists and without any neurological input, and this concept was soon abandoned. ${ }^{2}$ In 1972 after a painstaking case records evaluation of 1117 patients hospitalized in Pittsburgh for 'cerebrovascular disease' the investigators concluded that data from medical records for these patients was unreliable, and 'treatment', (what there was), remained uneven and unproven. They concluded that a major impediment to obtaining reliable data was that stroke was 'considered a hopeless disease', its investigation largely neglected, an unattractive and undesirable cinderella amongst other vascular diseases. $^{3}$

The first positive therapeutic note followed the establishment of a Neuro Vascular Care Unit (NICU) in San Francisco in 1970, where a 'before-after' analysis compared the results of NICU treatment to standard 'community' hospital care. The authors found that the NICU group had a 50\% reduced complication rate. This was the first study to suggest a beneficial effect of stroke care attributable only to the special care stroke unit. ${ }^{4} \mathrm{~A}$ similar unit, established independently in Canada in 1975, with intensive care facilities including cardiac monitoring also found a beneficial effect on outcome in acutely admitted stroke patients, but like its predecessors, lacked a strictly comparable control group, and so was vulnerable to the criticism of referral bias on hospital admission. ${ }^{5}$ The scarcity of CT brain scanning, still not a routine procedure in stroke patients at this time, also hindered comparisons with patients treated in non-intensive care facilities.

Studies of non-intensive care populations, where the prime goal is rehabilitation, are not comparable to any of these studies, when brain scanning by CT or MRI is not standard, in view of the inaccuracy of stroke diagnosis on clinical grounds alone, even with experts in the field. For instance, in studies comparing the initial clinical diagnosis of stroke patients to the diagnosis after hospital investigation where brain scanning was routine, the accuracy was $<80 \%$, the most common misdiagnosis being post ictal states..$^{6,7}$

\section{Evolution of a scientific basis for stroke research}

These early intensive care units also formed a base on which to launch, for the first time, a systematic and scientific approach to investigation and research in all aspects of acute cerebrovascular disease in a clearly defined population, including the logistics of organization, relationship to rehabilitation, and nursing and medical care.

Computed tomography brain scanning gradually became an essential diagnostic criterion in stroke diagnosis by the late 1970s, since besides excluding other pathologies, before then the only way to differentiate cerebral hemorrhage from infarction was by lumbar puncture, a dangerous investigation of little value

From the Division of Clinical Neuroscience (JWN), St. George Medical School, University of London, London, England; University of Western Ontario (VH), University Hospital, London, Ontario, Canada.

Received December 5, 2008. Final Revisions Submitted December 10, 2008 Correspondence to: John W. Norris, Clinical Neuroscience, St. Georges Medical School, University of London, London, SW7 3NT, UK. 


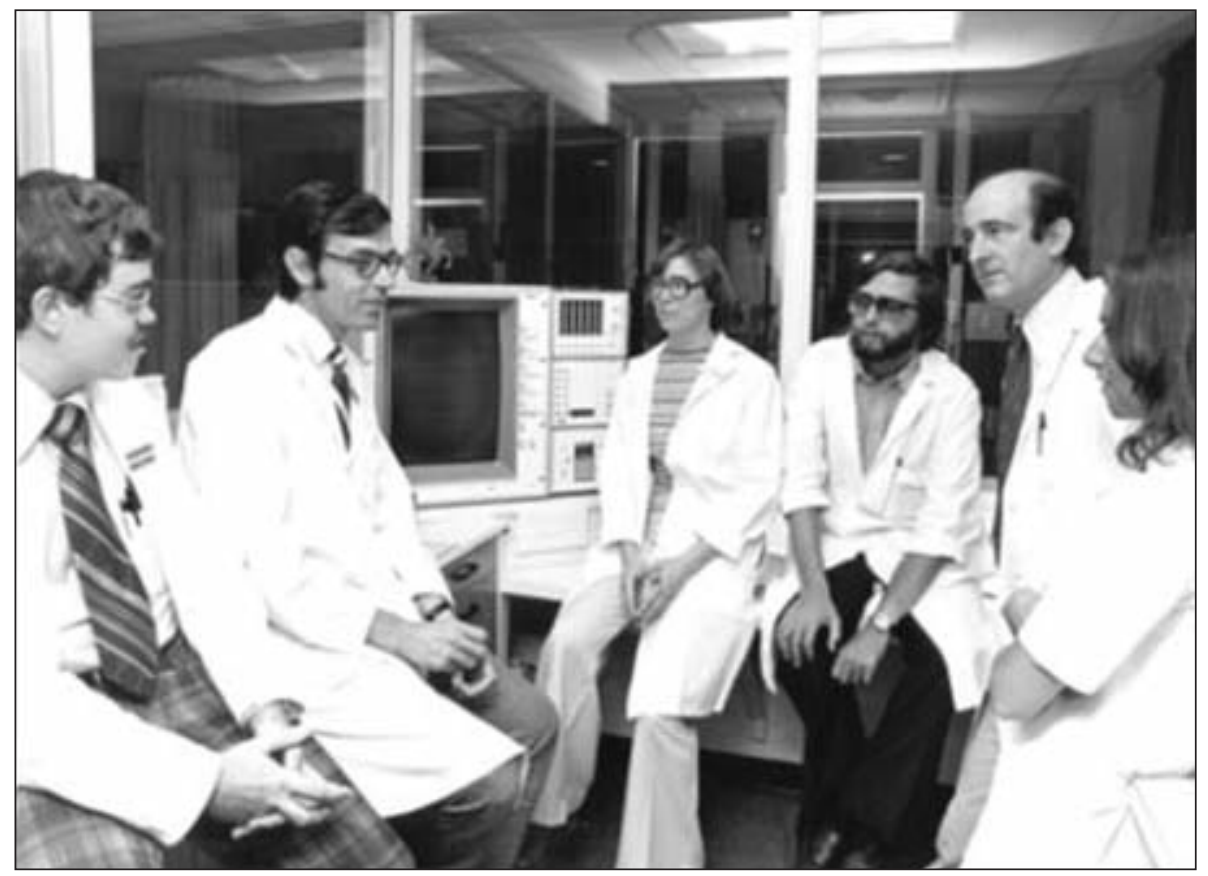

Figure: The Toronto Stroke Unit 1975.

in subsequent management. Isotopic brain scanning enjoyed transient popularity but added little valuable information and was soon abandoned.

Drug trials used methodology then that would be unacceptable today, such as in 1965, where a therapeutic trial of anticoagulants in 20 randomized patients was considered conclusive. ${ }^{8}$ Increasingly sophisticated statistical methodology soon developed using reliable evidence of pathology, paving the way for clinically valid drug studies in stroke prevention as well as acute care, and involved populations of patients, numbered in hundreds or even thousands, on which most present therapeutic strategies are based.

With the gradual spread worldwide of stroke units, and patients with reliable evidence of established cerebral pathology, numerous drug studies were then undertaken, both for acute treatment as well as for the secondary prevention of stroke. These included treatment of acute cerebral edema using corticosteroids, hypertonic solutions, hyperventilation and a great variety of so called neuroprotectant drugs for the very acute stages of cerebral infarction.

However, many drugs apparently effective in animal models, have not yet been found effective in humans, despite increasingly sophisticated knowledge of the physiology and cellular pathology of cerebral ischemia. ${ }^{9}$ Also, many of these early drugs were abandoned on what would be considered insufficient scientific evidence today, usually with patient numbers too small and drugs given too late, for any definitive conclusions. For instance, neither corticosteroid therapy nor intravenous hypertonic solutions have been tested properly using the stringent trial criteria needed in modern methodology.
Is intensive care of stroke a waste of time, money and resources?

Even 15 years after the first stroke ICU was opened in Pittsburgh, debate continued over its value for money. Studies of cardiac monitoring in stroke patients had indicated a variety of cardiac dysfunction, particularly dysrythmias, but this facility was found expensive and with little apparent impact on patients clinical outcome or recovery. ${ }^{10,11}$

A Swedish group initiated the first critical comparative study in 1985 of unselected acute stroke patients in a non-intensive stroke unit comparing their outcome to those admitted to a general medical ward in the same hospital. ${ }^{12}$ Case fatality rates were similar in the two groups but, by three months, only $15 \%$ of stroke unit patients were hospitalized compared to $39 \%$ in the general medical wards. The authors concluded that team work between all medical staff, including physicians, nursing and physical medicine improves functional outcome and reduces the need for long-term hospital care.

Following a similar study of randomly matched groups of 220 patients admitted to a stroke unit and compared to a general medical ward in Trondheim, Norway, in 1991, the authors concluded that acute care in a stroke unit was the most effective treatment that could be offered at that time. ${ }^{13}$ This was before the proven value of thrombolytic therapy, which was not established until the 1995 publication from the US National Institutes of Neurological Disorders. ${ }^{14}$

In a recent Cochrane review of organized inpatient stroke unit care, the authors compared stroke unit care to an 'alternative service' of 6936 patients in 31 published trials. ${ }^{15}$ They concluded 
that patients with acute stroke are more likely to survive, return home and regain independence if treated in a stroke unit. The reason for this therapeutic effect is still debated. It may be because of better diagnostic procedures, better nursing care, early mobilization, prevention of complications or more effective rehabilitation. These beneficial effects persist as long as ten years later ${ }^{16}$ but their effect is not as apparent in data from 'mobile stroke teams', stroke 'pathways' or other short cuts with more economy but little therapeutic impact.

\section{Future advances in stroke care}

The evolution of acute stroke care continues. The increasing evidence of the serious and imminent threat of stroke from transient ischemic attacks (TIAs) is forcing a new look at urgent assessment and prophylactic treatment of stroke within hours, not weeks or months. Patients with TIAs must be assessed urgently, and their acute care incorporated within the same therapeutic boundaries as the acute stroke patients. ${ }^{17}$ Acute stroke care units therefore need to incorporate acute assessment of TIA patients in the same location so that evaluation of cause and immediate management can be administered urgently.

It is not the intention of this article to duplicate the already existing comprehensive published evaluations of acute stroke care. Although the concepts of acute stroke care and the early attempts to implement it were initially a North American initiative in the late $1960 \mathrm{~s}$, the practical implementation, development and evaluation has been a largely European initiative, especially Scandinavian. Excellent comprehensive and topical reviews are already published ${ }^{15,18,19}$ to quote but a few. Although the concept of thrombolytic treatment of acute ischemic stroke was mooted 50 years ago ${ }^{20}$, its rediscovery, as an effective therapy half a century later, was a direct effect of the present day implementation of acute stroke units.

This review is a perspective of the enormous advances in medical care which have occurred, and are still occurring world wide, in stroke. At the same time, it highlights how much remains to be done. The World Stroke Day Proclamation which serves as a Global Stroke Agenda, points out in part, that "Organized stroke care improves outcomes, but remains the exception nearly everywhere". Stroke, the world's third most common cause of death and disability, neglected for so long, remains a "treatable and preventable catastrophe". ${ }^{21}$

\section{REFERENCES}

1. Kennedy FB, Pozen TJ, Gabelman GH, Tuthill JE, Zaentz SD. Stroke intensive care-an appraisal. Am Heart J. 1970; 80: 188-96.

2. Cooper SW, Olivet JA, Woolsey FM. Establishment and operation of combined intensive care unit. N Y State J Med. 1972; 72: 2215-20.

3. Carpenter RR, Reed DE. The outcome for patients with cerebrovascular disease in university and community hospitals. Stroke. 1972; 3:747-58

4. Drake WE, Hamilton MJ, Carlsson M, Blumenkrantz J. Acute stroke management and patient outcome: the value of acute neurovascular care units. Stroke. 1973; 4:933-45.

5. Norris JW, Hachinski VC. Intensive care management of stroke patients. Stroke. 1976; 7:573-7.

6. Norris JW, Hachinski VC. Misdiagnosis of stroke. Lancet. 1982; $1: 328-31$.

7. Harbinson J, Hossain O, Jenkinson D, Davis J. Diagnostic accuracy of stroke referrals from primary care, emergency room physicians, and ambulance staff using the face arm and leg speech test. Stroke. 2003; 34:71-6.

8. Pearce JM, Gubbay SS, Walton JM. Long term anticoagulant therapy in transient cerebral ischaemic attacks. Lancet. 1965; 1 : 6-9.

9. Fisher M. Advances in stroke 2007. Stroke. 2008; 39:250-1.

10. Lavy S, Yaar I, Melamed E, Stern S. Effect of acute stroke on cardiac functions as observed in an intensive care stroke unit. Stroke. 1974; 5:775-80.

11. Norris JW, Froggat GM, Hachinski VC. Cardiac arrhythmias in acute stroke. Stroke. 1978; 9:392-5.

12. Strand T, Asplund K, Eriksson S, Hagg E, Lithner F, Wester PO. A non intensive stroke unit reduces functional disability and the need for long term hospitalisation. Stroke. 1985; 16:29-34.

13. Indredavik B, Bakke F, Solberg R, Rokseth R, Haaheim L, Holme I. Benefit of a stroke unit: a randomised clinical trial. Stroke. 1991; 22:1026-31.

14. The National Institute of Neurological Disorders and Stroke: rt-PA stroke study group. Tissue plasminogen activator for acute ischemic stroke. N Engl J Med. 1995; 333:1581-2.

15. Govan L, Weir CJ, Langhorne P, for the Stroke Unit Trialists' Collaboration. Organised inpatient (stroke unit) care for stroke. Stroke. 2008; 39;2402-3.

16. Cochrane database of systematic reviews 2007 Issue 4. Art No. CD000197. DOI:10.1002/14651858. CD000197.pub2

17. Rothwell PM, Giles MF, Chandratheva A, Marquard L, Geraghty O, Redgrave $\mathrm{J}$, et al. Effect of urgent treatment of transient ischaemic attack and minor stroke on early recurrent stroke (EXPRESS Study): a prospective population - based sequential comparison. Lancet. 2007; 370:1432-42.

18. Norrving B, Adams RJ. Organised stroke care. Stroke. 2005; 37: 326-8.

19. Norrving B, Wester P, Sunnerhagen KS, Terent A, Sohlberg A, Berggren F. Beyond conventional stroke guidelines. Stroke. 2007; 38:2185-90.

20. Sussman B, Fitch T, Plainfield N. Thrombolysis with fibrinolysin in cerebral arterial occlusion. JAMA. 1958; 167:1705-9.

21. Hachinski V. World Stroke Day 2008. 'Little strokes, big trouble'. Stroke. 2008; 39:2407-8. 